\title{
Formulation of a New Implicit Method for Group Implicit BBDF in Solving Related Stiff Ordinary Differential Equations
}

\author{
Norshakila Abd Rasid $^{1,2}$, Zarina Bibi Ibrahim ${ }^{1, *}$, Zanariah Abdul Majid ${ }^{1}$, Fudziah Ismail ${ }^{1}$ \\ ${ }^{1}$ Department of Mathematics, Faculty of Science, Universiti Putra Malaysia, 43400 UPM, Serdang, Selangor, Malaysia \\ ${ }^{2}$ Universiti Kuala Lumpur, Malaysian Institute of Marine Engineering Technology (UniKL MIMET), 32200, Lumut, Perak, Malaysia
}

Received April 10, 2019; Revised January 20, 2020; Accepted March 16, 2021

\section{Cite This Paper in the following Citation Styles}

(a): [1] Norshakila Abd Rasid, Zarina Bibi Ibrahim, Zanariah Abdul Majid, Fudziah Ismail, "Formulation of a New Implicit Method for Group Implicit BBDF in Solving Related Stiff Ordinary Differential Equations," Mathematics and Statistics, Vol. 9, No. 2, pp. 144 - 150, 2021. DOI: 10.13189/ms.2021.090208.

(b): Norshakila Abd Rasid, Zarina Bibi Ibrahim, Zanariah Abdul Majid, Fudziah Ismail (2021). Formulation of a New Implicit Method for Group Implicit BBDF in Solving Related Stiff Ordinary Differential Equations. Mathematics and Statistics, 9(2), 144 - 150. DOI: 10.13189/ms.2021.090208.

Copyright $(2021$ by authors, all rights reserved. Authors agree that this article remains permanently open access under the terms of the Creative Commons Attribution License 4.0 International License

\begin{abstract}
This paper proposed a new alternative approach of the implicit diagonal block backward differentiation formula (BBDF) to solve linear and nonlinear first-order stiff ordinary differential equations (ODEs). We generate the solver by manipulating the numbers of back values to achieve a higher-order possible using the interpolation procedure. The algorithm is developed and implemented in $\mathrm{C}++$ medium. The numerical integrator approximates few solution points concurrently with off-step points in a block scheme over a non-overlapping solution interval at a single iteration. The lower triangular matrix form of the implicit diagonal causes fewer differentiation coefficients and ultimately reduces the execution time during running codes. We choose two intermediate points as off-step points appropriately, which are proven to guarantee the method's zero stability. The off-step points help to increase the accuracy by optimizing the local truncation error. The proposed solver satisfied theoretical consistency and zero-stable requirements, leading to a convergent multistep method with third algebraic order. We used the well-known and standard linear and nonlinear stiff IVP problems used in literature for validation to measure the algorithm's accuracy and processor time efficiency. The performance metrics are validated by comparing them with a proven solver, and the output shows that the alternative method is better than the existing one.
\end{abstract}

Keywords Implicit Method, Off-Step, Stiff Problems

\section{Introduction}

In applied science and engineering problems, the inclusion of ordinary differential equations (ODEs) in a dynamic model is vital in demonstrating the state of the system. The higher-order and complex phenomena of ODEs barely have analytical solutions, and it advocates using numerical methods to approximate the solutions for the problems. Frequent dynamic ODE models are governed by the unique behavior identified as stiffness. The stiffness caused the solutions to become unbearably slow, and only a few ODEs integration methods can be applied in all spatial locations. On top of that, a wide range of stiffness may exist in the standard practice set of ODEs. Thus, selecting low-cost and economical numerical methods is urging based on local conditions. Although stiff ODEs are the matured area in practices, the need to produce quality and improved methods in line with the systems' demand and difficulty cannot cease [1].

Our primary interest in this paper is solving an initial value problem (IVP) of ordinary differential equations (ODEs) of order 2 (two), which can be expressed:

$$
y^{\prime}=F(x, y(x)), y(a)=y_{0}, x \in[a, b]
$$


The equation (1) known as linear ODEs with constant coefficient if $F(x, y(x))=P(x) y+Q(x)$ with solutions of the form $y(x)=\sum_{t=1}^{m} C_{t} e^{-\lambda_{t} x}+\vartheta(x)$. The notations for

$y(x), F, \eta$, and $Q(x)$ are in the form of $r \times 1$ column vector where $P(x)$ is a $r \times r$ non-singular matrix. Meanwhile, $\lambda_{i}$, where $i=1,2, \ldots, m$ are defined as the eigenvalues of the Jacobian matrix $P(x)$ The problem (1) always has a unique solution [2].

According to [1], stiffness features are classified through the eigenvalues of the Jacobian matrix that exist in the left half-plane of the complex plane, alternatively stated that the $\lambda_{i}$ must have a maximum negative magnitude. The second condition for stiffness is outlined by the absolute value of the stiffness ratio between the maximum and minimum of eigenvalues that must contain maximum negative values.

Stiff ODEs produced a part of solutions called the transient phase. The solution rapidly decays zero as $t$ increases and requires the stability equation to take a smaller step size. It became impracticable for forwarding step methods [3].

As reported by [1], the implicit methods are significantly fitted in solving stiff ODEs because of their higher-order accuracy as compared to explicit ones. However, [4] set up the limitation for stability and proved that stable multistep-method did not exceed order over two when solving stiff ODEs. The alternative approaches in [5] and [6] had proposed to overcome the limitation. These approaches involved modifications of the multistep method procedure for achieving a higher-order and stable method. In 1964, [7] introduced a hybrid multistep method for the IVP to overcome the Dalquist's barrier in [4]. The hybrid terms classified as $h \beta f_{n-\theta}$ where $\theta=1 / 2$ or $1 / 3$. in the method and provided stable and convergent solutions. Besides, [8] proposed a strategy to improve the accuracy and order of the methods by adding the off-step points and future points. Relevant research on the hybrid multistep methods can be found in [9]-[11].

In 1952, [12] had overcome stiffness in their kinetic studies and stated that backward differentiation formula (BDF) was the best method for solving stiff ODEs. [13] improvised the BDF method with the new approach of calculating a few solution points simultaneously in a block at each iteration of the algorithm. It was identified as the block backward differentiation formula (BBDF) and produced the solutions with minimal time cost and lessened the number of integration steps. Since then, the study on BBDF captivated the attention and grew in numbers as seen in [15]-[19]. In recent studies by [17-18], a new approach of BBDF method proved that diagonally implicit block has effectively solved stiff ODEs.

The alternative formula introduced in this paper is a combination of diagonally implicit block BDF and off-step points. We simply call the formula DI2OBBDF. We present the formulation of the coefficients for
DI2OBBD in the next section.

\section{Materials and Methods}

\subsection{Derivation of the Method}

This section presented the derivation of the coefficients for DI2OBBDF by using Lagrange Interpolation Polynomial.

$$
P(x)=\sum_{s=0}^{m} \prod_{\substack{r=0 \\ s \neq r}}^{m} \frac{\left(x-x_{n+2-r}\right)}{\left(x_{n+2-s}-x_{n+2-r}\right)} y\left(x_{n+2-s}\right)
$$

Where $s=0, \frac{1}{2}, \ldots, m$.

According to [17] and [18], the derivation for diagonal implicit methods by using (2) is done sequentially. Thus, we calculated the coefficients for the points $y_{n+1 / 2}, y_{n+1}, y_{n+3 / 2}$ and $y_{n+2}$ separately. The formula for the first point, $y_{n+1 / 2}$ is produced by polynomial interpolation of three back values at points $\left(x_{n-2}, y_{n-2}\right),\left(x_{n-1}, y_{n-1}\right)$ and $\left(x_{n}, y_{n}\right)\left(x_{n}, y_{n}\right)$.

$$
\begin{gathered}
y_{n+1 / 2}(x)=\left(\frac{\left(x-x_{n-1}\right) \ldots\left(x-x_{n+1 / 2}\right)}{\left(x_{n-2}-x_{n-1}\right) \ldots\left(x_{n-2}-x_{n+1 / 2}\right)}\right) y_{n-2}+ \\
\ldots+\left(\frac{\left(x-x_{n-2}\right) \ldots\left(x-x_{n}\right)}{\left(x_{n+1 / 2}-x_{n-2}\right) \ldots\left(x_{n+1 / 2}-x_{n}\right)}\right) y_{n+1 / 2}
\end{gathered}
$$

For second point, $y_{n+1}$, the interpolating points consists of $x_{n-2}, x_{n-1}, x_{n}, x_{n+1 / 2}$ and $x_{n+1}$ are as follows:

$$
\begin{gathered}
y_{n+1}(x)=\left(\frac{\left(x-x_{n-1}\right) \ldots\left(x-x_{n+1}\right)}{\left(x_{n-2}-x_{n-1}\right) \ldots\left(x_{n-2}-x_{n+1}\right)}\right) y_{n-2}+ \\
\ldots+\left(\frac{\left(x-x_{n-2}\right) \ldots\left(x-x_{n+1 / 2}\right)}{\left(x_{n+1}-x_{n-2}\right) \ldots\left(x_{n+1}-x_{n}\right)}\right) y_{n+1}
\end{gathered}
$$

For the third point of the method $y_{n+3 / 2}$, (2) interpolates $x_{n-2}, x_{n-1}, x_{n}, x_{n+1 / 2}, x_{n+1}$ and $x_{n+3 / 2}$ as follows:

$$
\begin{aligned}
y_{n+3 / 2}(x)= & \left(\frac{\left(x-x_{n-1}\right) \ldots\left(x-x_{n+3 / 2}\right)}{\left(x_{n-2}-x_{n-1}\right) \ldots\left(x_{n-2}-x_{n+3 / 2}\right)}\right) y_{n-2}+ \\
& \ldots+\left(\frac{\left(x-x_{n-2}\right) \ldots\left(x-x_{n+1}\right)}{\left(x_{n+3 / 2}-x_{n-2}\right) \ldots\left(x_{n+3 / 2}-x_{n}\right)}\right) y_{n+3 / 2}
\end{aligned}
$$

The last point $y_{n+2}$ of formula is obtained by interpolates the points comprise $x_{n-2}, x_{n-1}, x_{n}, x_{n+1 / 2}, x_{n+1}, x_{n+3 / 2}$ and $x_{n+2}$

$$
\begin{aligned}
y_{n+3 / 2}(x)= & \left(\frac{\left(x-x_{n-1}\right) \ldots\left(x-x_{n+2}\right)}{\left(x_{n-2}-x_{n-1}\right) \ldots\left(x_{n-2}-x_{n+3 / 2}\right)}\right) y_{n-2}+ \\
& \ldots+\left(\frac{\left(x-x_{n-2}\right) \ldots\left(x-x_{n+1}\right)}{\left(x_{n+3 / 2}-x_{n-2}\right) \ldots\left(x_{n+3 / 2}-x_{n}\right)}\right) y_{n+3 / 2}
\end{aligned}
$$

The next step is to introduce the variable substitution $x=x_{n+1 / 2}+$ sh into equations (3), (4), (5) and (6) 
respectively. Then, we differentiate once for (3), (4), (5) and (6) with respect to s, following with substitution each formula with $s=0,1 / 2,1,3 / 2$ respectively, and the corrector formula is as follows:

$$
\begin{aligned}
y_{n+1 / 2}= & \frac{9}{184} y_{n-2}-\frac{25}{92} y_{n-1}+\frac{225}{115} y_{n}+\frac{15}{46} h f_{n+1 / 2} \\
y_{n+1}= & -\frac{2}{115} y_{n-2}+\frac{3}{23} y_{n-1}-\frac{18}{23} y_{n}+\frac{192}{115} y_{n+1 / 2}+\frac{6}{23} h f_{n+1} \\
y_{n+3 / 2}= & \frac{15}{1828} y_{n-2}-\frac{147}{1828} y_{n-1}+\frac{1225}{1828} y_{n}-\frac{735}{457} y_{n+1 / 2} \\
& +\frac{3675}{1828} y_{n+1}+\frac{105}{457} h f_{n+3 / 2} \\
y_{n+2}= & -\frac{3}{665} y_{n-2}+\frac{16}{285} y_{n-1}-\frac{12}{19} y_{n}+\frac{512}{285} y_{n+1 / 2}-\frac{48}{19} y_{n+1} \\
& +\frac{1536}{665} y_{n+3 / 2}+\frac{4}{19} h f_{n+2} .
\end{aligned}
$$

We stored the coefficients of DI2OBBDF in the algorithm to avoid the former tedious computation of the divided difference. We implemented the algorithm in PECE mode where $\mathrm{P}$ stands for predictor, $\mathrm{E}$ for evaluation of (1) and $C$ for corrector in (7). The same technique in (2) is applied for the derivation of predictor formula. The interpolation points involve $x_{n-1}, x_{n-1 / 2}$, and $x_{n}$.

$$
\begin{gathered}
y_{n+k}^{P}(x)=\left(\frac{\left(x-x_{n-1 / 2}\right)\left(x-x_{n}\right)}{\left(x_{n-1}-x_{n-1 / 2}\right)\left(x_{n-1}-x_{n}\right)}\right) y_{n-1} \\
+\left(\frac{\left(x-x_{n-1}\right)\left(x-x_{n}\right)}{\left(x_{n-1 / 2}-x_{n-1}\right)\left(x_{n-1 / 2}-x_{n}\right)}\right) y_{n+1 / 2} \\
+\left(\frac{\left(x-x_{n-1}\right)\left(x-x_{n-1 / 2}\right)}{\left(x_{n}-x_{n-1}\right)\left(x_{n}-x_{n-1 / 2}\right)}\right) y_{n}
\end{gathered}
$$

Replacing $x=x_{n+1}+$ sh, then, substituted the values of $s=-1 / 2,0,1 / 2,1$ to generate the following predictor formula for DI2OBBD:

$$
\begin{aligned}
& y_{n+1 / 2}=y_{n-1}-3 y_{n-1 / 2}+3 y_{n} \\
& y_{n+1}=3 y_{n-1}-8 y_{n-1 / 2}+6 y_{n+1 / 2} \\
& y_{n+3 / 2}=6 y_{n-1}-15 y_{n-1 / 2}+10 y_{n+1 / 2} \\
& y_{n+2}=10 y_{n-1}-24 y_{n-1 / 2}+15 y_{n}
\end{aligned}
$$

\subsection{Order of the Method}

This section addressed the calculation of an order for DI2OBBDF in (7) by referring to several definitions in [1]. Beforehand, the DI2OBBDF can be written in the following matrix form:

$$
\sum_{r=0}^{m} A_{r} Y_{n-2+r}=h \sum_{r=0}^{m} \beta_{r} Y_{n+2-r}^{\prime}
$$

where $A_{r}$ and $B_{r}$ are matrices of dimension $4 \times 4$. The functions $Y_{n-2+r}$ and $Y_{n-2+r}^{\prime}$ are the dimension 4 by 1 matrices and deduced by

$$
\begin{aligned}
& Y_{n-2+r} \\
& =\left[Y_{(n-2+r)+1 / 2}, Y_{(n-2+r)+1}, Y_{(n-2+r)+3 / 2}, Y_{(n-2+r)+2}\right]^{T} \\
& {\left[Y_{(n-2+r)+1 / 2}^{\prime}, Y_{(n-2+r)+1}^{\prime}, Y_{(n-2+r)+3 / 2}^{\prime}, Y_{(n-2+r)+2}^{\prime}\right]^{T}(11)}
\end{aligned}
$$

The corrector formula in (7) associated with linear operator can be written as follows:

$L[y(x), h]=\left[\sum_{i=0}^{k} A_{r} Y(x+i h / 2)-h \sum_{i=0}^{k} \beta_{r} Y^{\prime}(x+i h / 2)\right]$

Taylor's series are used for expanding the function $Y(x+i h / 2)$ and its derivative $Y^{\prime}(x+i h / 2)$ around $x$ :

$$
\begin{aligned}
Y(x+i h / 2)= & Y(x)+(i h / 2) Y^{\prime}(x)+(i h / 2)^{2} Y^{\prime \prime}(x) \\
& +(i h / 2)^{3} Y^{\prime \prime \prime}(x)+\ldots \\
Y^{\prime}(x+i h / 2) & =Y^{\prime}(x)+(i h / 2) Y^{\prime \prime}(x)+(i h / 2)^{2} Y^{\prime \prime \prime}(x) \\
& +(i h / 2)^{3} Y^{(4)}(x)+\ldots
\end{aligned}
$$

Substituting (13) into (12) gives

$$
\begin{aligned}
& L[y(x), h]=\left[\begin{array}{c}
A_{r}\left(\begin{array}{l}
Y(x)+(i h / 2) Y^{\prime}(x)+(i h / 2)^{2} Y^{\prime \prime}(x) \\
+(i h / 2)^{3} Y^{\prime \prime \prime}(x)
\end{array}\right) \\
\sum_{i=0}^{k}-h \sum_{i=0}^{k} \beta_{r}\left(\begin{array}{l}
Y^{\prime}(x)+(i h / 2) Y^{\prime \prime}(x) \\
+(i h / 2)^{2} Y^{\prime \prime \prime}(x) \\
+(i h / 2)^{3} Y^{(4)}(x)+\ldots
\end{array}\right)
\end{array}\right] \\
& L[y(x), h]=D_{0} Y(x)+D_{1} Y^{\prime}(x)+D_{2} Y^{\prime \prime}(x)+D_{3} Y^{\prime \prime \prime}(x)+\ldots
\end{aligned}
$$

The equations (14) is claimed to have order $\rho$ if $D_{0}=D_{1}=D_{2}=\ldots=D_{\rho}=0$ and $D_{\rho+1} \neq 0$.

Thus, we determine the order for the proposed method equal to:

$$
D_{4}=\left[-\frac{75}{184}, 0,0,0\right]^{T}
$$

Thus, we get $D_{4} \neq 0$. Hence, the corrector method (7) is an order three method.

\subsection{Convergent of the Method}

The following theorem stated in [1] defines the convergent criteria for DI2OBBDF.

Theorem 1. The necessary and sufficient conditions for the linear multistep method (LMM) of (10) to be convergent are that it must be consistent and zero stable.

The terms $\omega_{r}$ and $\zeta_{r}$ where $r=1,2, \ldots, 11$. are used to classify the elements of the matrix in (7) as follows: 


$$
\begin{aligned}
& \omega_{1}=\omega_{2}=\omega_{4}=\omega_{6}=\left[\begin{array}{llll}
0 & 0 & 0 & 0
\end{array}\right]^{T} \\
& \omega_{3}=\left[\begin{array}{llll}
\frac{9}{184} & -\frac{2}{115} & \frac{15}{1828} & -\frac{3}{665}
\end{array}\right]^{T} \\
& \omega_{5}=\left[\begin{array}{llll}
-\frac{25}{92} & \frac{3}{23} & -\frac{147}{1828} & \frac{16}{285}
\end{array}\right]^{T} \\
& \omega_{7}=\left[\begin{array}{llll}
\frac{225}{115} & -\frac{18}{23} & \frac{1225}{1828} & -\frac{12}{19}
\end{array}\right]^{T} \\
& \omega_{8}=\left[\begin{array}{llll}
1 & \frac{192}{115} & -\frac{735}{457} & \frac{512}{285}
\end{array}\right]^{T} \\
& \omega_{9}=\left[\begin{array}{llll}
0 & 1 & \frac{3675}{1828} & -\frac{48}{19}
\end{array}\right]^{T} \\
& \omega_{10}=\left[\begin{array}{llll}
0 & 0 & 1 & \frac{1536}{665}
\end{array}\right]^{T} \\
& \omega_{10}=\left[\begin{array}{llll}
0 & 0 & 0 & 1
\end{array}\right]^{T} \\
& \zeta_{1}=\ldots=\zeta_{7}=\left[\begin{array}{llll}
0 & 0 & 0 & 1
\end{array}\right]^{T} \\
& \zeta_{8}=\left[\begin{array}{llll}
\frac{15}{46} & 0 & 0 & 0
\end{array}\right]^{T} \\
& \zeta_{9}=\left[\begin{array}{llll}
0 & \frac{6}{23} & 0 & 0
\end{array}\right]^{T} \\
& \zeta_{10}=\left[\begin{array}{llll}
0 & 0 & \frac{105}{457} & 0
\end{array}\right]^{T} \\
& \zeta_{11}=\left[\begin{array}{llll}
0 & 0 & 0 & \frac{4}{19}
\end{array}\right]^{T}
\end{aligned}
$$

The following conditions elaborate the criteria for consistency of the DI2OBBDF

Definition 1. A LMM in (10) is said to be consistent if it has the order $\boldsymbol{\rho} \geq \mathbf{1}$, if only it satisfies the following condition:

1. $\begin{gathered}\sum_{r=0}^{11} \omega_{r}=\omega_{0}+\omega_{1}+\omega_{2}+\omega_{3}+\omega_{4}+\omega_{5}+\omega_{6} \\ \omega_{7}+\omega_{8}+\omega_{9}+\omega_{10}+\omega_{11}=\left[\begin{array}{llll}0 & 0 & 0 & 0\end{array}\right]^{T}\end{gathered}$.

$$
\sum_{r=0}^{11} r \omega_{r}=0 \omega_{0}+1 \omega_{1}+2 \omega_{2}+3 \omega_{3}+4 \omega_{4}+5 \omega_{5}+6 \omega_{6}
$$

2. $7 \omega_{7}+8 \omega_{8}+9 \omega_{9}+10 \omega_{10}+11 \omega_{11}$ $=\sum_{r=0}^{11} 2 \zeta_{r}=2 \zeta_{8}+2 \zeta_{9}+2 \zeta_{10}+2 \zeta_{11}$.
Thus, DI2OBBDF in (7) is consistent.

Theorem 2. The necessary and sufficient condition for the method given by (10) to be zero stable is that it satisfies the root condition. [1]

We associated the DI2OBBDF with the scalar test equation, $y^{\prime}(x)=\lambda y(x)$ with notation $H=h \lambda$ for verification of root condition. We solved the formula by the determinant of the matrix and obtain the sets of roots that satisfied $\left|v_{i}\right| \leq 1$

Hence, DI2OBBDF satisfied the criteria of a convergent method.

\section{Numerical Result}

This section demonstrated the efficiency of the derived method by comparing it with a proven solver, implicit 2-point block BDF (BBDF) in [13]. We validate the derived method's performance via test problems that consist of linear and nonlinear stiff problems. The problems are listed as follows and are taken from [17]:

Problem 1 (Linear)

$$
\begin{aligned}
& y^{\prime}=\xi(x-y)+1, \quad y(0)=1, \quad x \in[0,10], \\
& \xi=100 . \text { Solution: } y(x)=e^{-\xi x}+x .
\end{aligned}
$$

Problem 2 (Linear)

$$
\begin{aligned}
& y_{1}^{\prime}=(\xi+98) y_{1}+(\xi+99) y_{2}, \quad y_{1}(0)=1, \quad x \in[0,10], \\
& y_{2}^{\prime}=-(\xi+298) y_{1}-(\xi+299) y_{2}, y_{2}(0)=-1, \\
& \xi=100 . \text { Solutions: } y_{1}(x)=e^{-x}, y_{2}(x)=-e^{-x} .
\end{aligned}
$$

Problem 3 (Non-linear)

$$
\begin{aligned}
& y^{\prime}=-y^{3} / 2, \quad y(0)=1, \quad x \in[0,4] . \\
& \text { Solution: } y(x)=1 /(\sqrt{1+x}) .
\end{aligned}
$$

Problem 4 (Non-linear)

$$
\begin{aligned}
& y^{\prime}=(50 / y)-50 y, \quad y(0)=\sqrt{2}, \quad 0 \leq x \leq 1 . \\
& \text { Solution: } y(x)=\sqrt{1+e^{-100 x}} .
\end{aligned}
$$

We validate the efficiency of the proposed method with respect to maximum error in (16), and execution time with fixed step-size, $h$. We define the notations used in the table as follows:
$\mathrm{h}$
MAXE
: Step size.
TIME
: Maximum error.
DI2OBBDF
: CPU times in microseconds.
BBDF
: Diagonally Implicit Two-point
BBDF with two off-step points..
: Implicit BBDF [13] 
Computation of maximum error is defined as

$$
M A X E=\max _{1 \leq i \leq N S}\left(\left|y_{\text {exact }}^{(i)}-y_{\text {approximate }}^{(i)}\right|\right)
$$

Numerical result is as follows: The accuracy and execution time of the methods are presented in Tables 1-4. In conjunction, we plotted $\log _{10}(M A X E)$ $\log _{10}$ (MAXE) versus $\log _{10}(T I M E)$ graphs for each problem in Figures 1-4 to support the result obtained.

Table 1. Numerical result for Problem 1.

\begin{tabular}{|c|c|c|c|}
\hline $\mathrm{h}$ & Methods & MAXE & TIME \\
\hline \multirow{2}{*}{$10^{-2}$} & DI2OBBDF & $2.81426 \mathrm{e}-02$ & 124 \\
& BBDF & $1.83156 \mathrm{e}-02$ & 162 \\
\hline \multirow{2}{*}{$10^{-3}$} & DI2OBBDF & $5.12369 \mathrm{e}-03$ & 247 \\
& BBDF & $1.15700 \mathrm{e}-02$ & 671 \\
\hline \multirow{2}{*}{$10^{-4}$} & DI2OBBDF & $6.52934 \mathrm{e}-05$ & 459 \\
& BBDF & $1.55714 \mathrm{e}-04$ & 966 \\
\hline \multirow{2}{*}{$10^{-5}$} & DI2OBBDF & $6.71575 \mathrm{e}-07$ & 3610 \\
& BBDF & $1.60347 \mathrm{e}-06$ & 6187 \\
\hline \multirow{2}{*}{$10^{-6}$} & DI2OBBDF & $6.73466 \mathrm{e}-09$ & 4537 \\
& BBDF & $1.60817 \mathrm{e}-08$ & 71674 \\
\hline
\end{tabular}

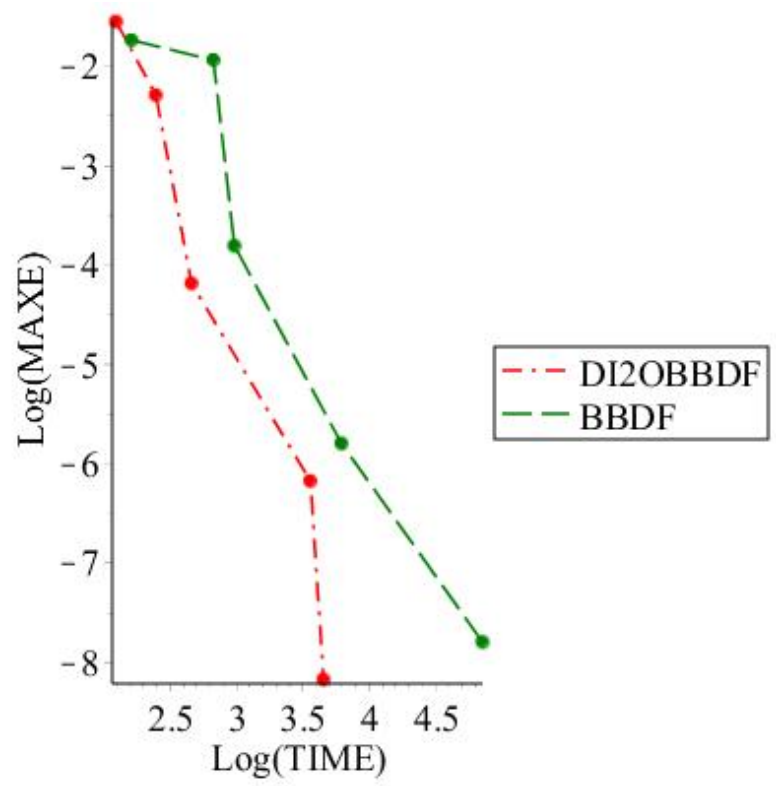

Figure 1. Accuracy curve for Problem 1

Table 2. Numerical result for Problem 2.

\begin{tabular}{|c|c|c|c|}
\hline $\mathrm{h}$ & Methods & MAXE & TIME \\
\hline \multirow{2}{*}{$10^{-2}$} & DI2OBBDF & $4.03031 \mathrm{E}-05$ & 50 \\
& BBDF & $6.85453 \mathrm{E}-02$ & 86 \\
\hline \multirow{2}{*}{$10^{-3}$} & DI2OBBDF & $4.09940 \mathrm{E}-07$ & 79 \\
& BBDF & $7.33973 \mathrm{E}-04$ & 122 \\
\hline \multirow{2}{*}{$10^{-4}$} & DI2OBBDF & $4.10637 \mathrm{E}-09$ & 423 \\
& BBDF & $7.35580 \mathrm{E}-05$ & 867 \\
\hline \multirow{2}{*}{$10^{-5}$} & DI2OBBDF & $4.10711 \mathrm{E}-11$ & 4141 \\
& BBDF & $7.35741 \mathrm{E}-06$ & 8599 \\
\hline \multirow{2}{*}{$10^{-6}$} & DI2OBBDF & $7.87898 \mathrm{E}-11$ & 49532 \\
& BBDF & $7.35765 \mathrm{E}-07$ & 70383 \\
\hline
\end{tabular}

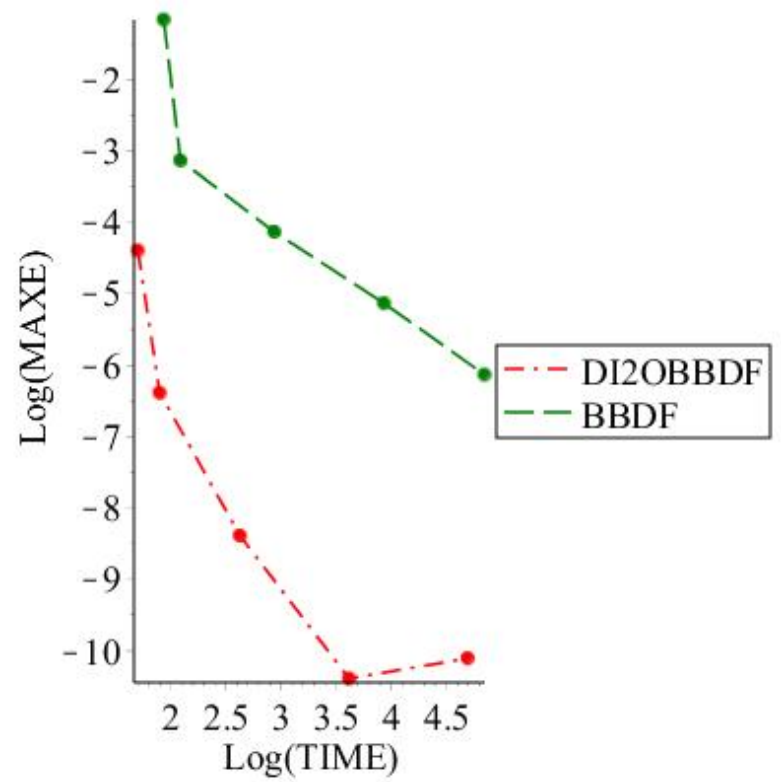

Figure 2. Accuracy curve for Problem 2.

Table 3. Numerical result for Problem 3.

\begin{tabular}{|c|c|c|c|}
\hline $\mathrm{h}$ & Methods & MAXE & TIME \\
\hline \multirow{2}{*}{$10^{-2}$} & DI2OBBDF & $2.97983 \mathrm{E}-05$ & 54 \\
& BBDF & $3.53439 \mathrm{E}-03$ & 78 \\
\hline \multirow{2}{*}{$10^{-3}$} & DI2OBBDF & $3.07008 \mathrm{E}-07$ & 61 \\
& BBDF & $3.66407 \mathrm{E}-04$ & 294 \\
\hline \multirow{2}{*}{$10^{-4}$} & DI2OBBDF & $3.07933 \mathrm{E}-09$ & 314 \\
& BBDF & $3.67732 \mathrm{E}-05$ & 933 \\
\hline \multirow{2}{*}{$10^{-5}$} & DI2OBBDF & $4.12150 \mathrm{E}-11$ & 1646 \\
& BBDF & $3.67865 \mathrm{E}-06$ & 22826 \\
\hline \multirow{2}{*}{$10^{-6}$} & DI2OBBDF & $2.22013 \mathrm{E}-10$ & 13361 \\
& BBDF & $3.67888 \mathrm{E}-07$ & 178132 \\
\hline
\end{tabular}

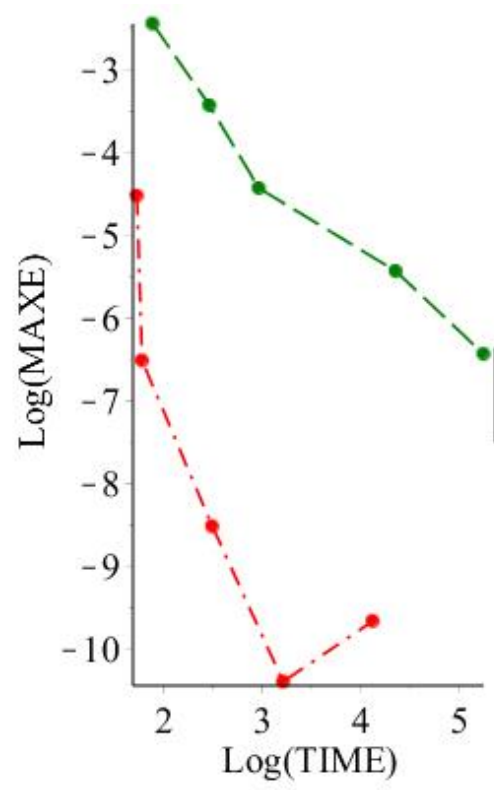

Figure 3. Accuracy curve for Problem 3. 
Table 4. Numerical result for Problem 4.

\begin{tabular}{|c|c|c|c|}
\hline $\mathrm{h}$ & Methods & MAXE & TIME \\
\hline \multirow{2}{*}{$10^{-2}$} & DI2OBBDF & $3.06559 \mathrm{E}-02$ & 71 \\
& BBDF & $2.60154 \mathrm{E}-01$ & 108 \\
\hline \multirow{2}{*}{$10^{-3}$} & DI2OBBDF & $9.72242 \mathrm{E}-04$ & 86 \\
& BBDF & $2.13934 \mathrm{E}-02$ & 256 \\
\hline \multirow{2}{*}{$10^{-4}$} & DI2OBBDF & $1.07465 \mathrm{E}-05$ & 151 \\
& BBDF & $2.55561 \mathrm{E}-03$ & 1679 \\
\hline \multirow{2}{*}{$10^{-5}$} & DI2OBBDF & $1.08765 \mathrm{E}-07$ & 872 \\
& BBDF & $2.59674 \mathrm{E}-04$ & 7378 \\
\hline \multirow{2}{*}{$10^{-6}$} & DI2OBBDF & $1.09202 \mathrm{E}-09$ & 3016 \\
& BBDF & $2.60084 \mathrm{E}-05$ & 11590 \\
\hline
\end{tabular}

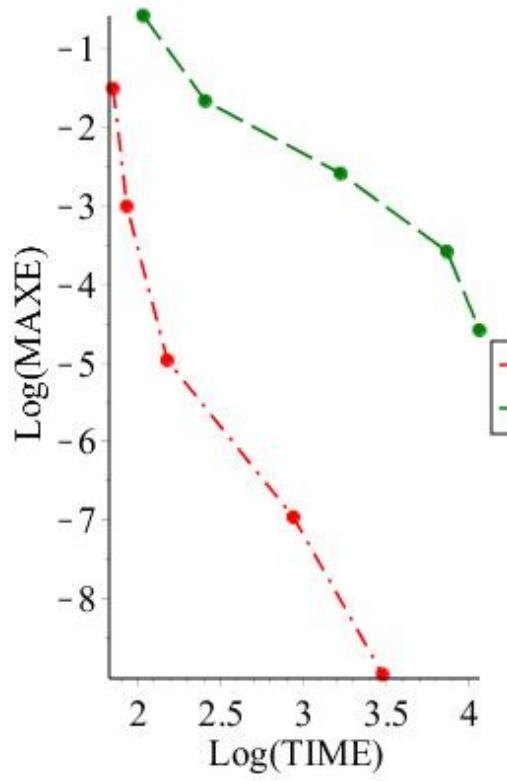

Figure 4. Accuracy curve for Problem 4.

In Figure 1-4, we plotted the accuracy curves for the DI2OBBDF and BBDF. The figure depicts the scale of Log (MAXE) versus Log (TOL) for each solver, and the small magnitude ordinates indicate the minimum error and the faster the time for running code. Based on the result, DI2OBBDF converged fastest for all test problems than other proven solvers since the growth of error curves in figures was smaller in values and shorter in the distance.

\section{Conclusion}

We constructed an implicit diagonal BBDF with off-step points to accurately approximate linear and nonlinear stiff IVP solutions. The analysis through the order, consistency, and zero stability resulted in a convergent method. We found that the significant contribution of DI2OBBDF is the ability to produce multiple solutions in one iteration. Meanwhile, the implicit diagonal form of DI2OBBDF led to Jacobian Newton's coefficients matrix in a lower triangular form, thus shortening the time required to run the codes. The presence of off-step points optimized the local error by increasing the accuracy. The numerical outputs of four test problems in Section 5 demonstrate that DI2OBBDF is a powerful tool for solving linear and nonlinear ODEs.

Therefore, DI2OBBDF is significantly useful for efficient approximations in a broad scope of ODEs in engineering and science.

\section{Acknowledgements}

This research was supported by Universiti Putra Malaysia under Grant Putra Scheme (GP/2018/9650600).

\section{REFERENCES}

[1] J. D. Lambert, Computational Methods in Ordinary Differential Equations, John Wiley and Sons, Chichester, New York. 1973.

[2] P. Henrici, Discrete variable methods in ordinary differential equations, John Wiley and Sons, New York, NY, USA, 1962.

[3] B. L. Ehle, A Comparison of Numerical Methods for Solving Certain Stiff Ordinary Differential Equations. Dept. of Math. University of Victoria, 1972, Report No. 70

[4] G. A. Dahlquist, Special Stability Problem for Linear Multistep Method, BIT, Vol. 3, pp. 27-43 1963.

[5] W. B. Gragg, H. J. Stetter, Generalized Multistep Predictor Corrector Methods, J of the Assoc for Comp Machinery, Vol. 11, No. 2, pp.188-209. 1964.

[6] J. C. Butcher, A Modified Multistep Method for the Numerical Integration of Ordinary Differential Equations, J of the Assoc for Comp Machinery, Vol. 12, No. 1, pp.124-135. 1965

[7] C. W. Gear, Hybrid Methods for Initial Value Problems in Ordinary Differential Equations, J. SIAM Numer.Anal. Scr. B, Vol. 2, No. 1, 1964.

[8] J. C. Butcher, A Modified Multistep Method for the Numerical Integration of Ordinary Differential Equations, J of the Assoc for Comp Machinery, Vol.12, No.1, pp124-1351965.

[9] A. K. Ezzesdine, Hybrid Extended Backward Differentiation Formula For Stiff system, Int. J. of Nonlinear Sciences, Vol. 2, pp196-204 2011.

[10] M. Ebadi, M. Y. Gokhale, Hybrid BDF Method for the numerical solutions of ODEs, Numer. Algo, Vol. 55, pp. $1-17,2009$.

[11] N. Abasi, 2- Point Block BDF Method with Off-Step Points for Solving Stiff ODEs, Journal of Soft Computing and Application, vol. 2014, Article ID jsca-00039, 2014.

[12] C. Curtiss, J. O. Hirschfelder, Integration of stiff equations, Proceeding of the National Academy of Sciences of the United State of America, Vol. 38, pp235-243 1952.

[13] Z. B. Ibrahim, K. I. Othman and M. B. Suleiman. Implicit r-Point Block Backward Differentiation Formula for solving 
First-Order Stiff ODEs, Applied Mathematics and Computation, vol. 186, no. 1, pp. $558-565.2007$.

[14] Z. B. Ibrahim, M. B. Suleiman and N. A. A. Nasir. Convergence of the 2-Point Block Backward Differentiation Formulas, Applied Mathematical Sciences, vol. 5, no. 70, pp. 3473-3480. 2011

[15] N. A. A. Nasir, Z. B. Ibrahim, K. I. Othman and M. B. Suleiman. Fifth Order Two-Point Block Backward Differentiation Formulas for Solving Ordinary Differential Equations, Applied Mathematical Sciences, vol. 5, no. 69-72, pp. 3505-3518, 2011.

[16] I. S. M. Zawawi, Z. B. Ibrahim, F. Ismail and Z. A. Majid. Diagonally Implicit Block Backward Differentiation Formulas for Solving Ordinary Differential Equations, Internat. J. Math \& Math Sci, vol. 2012, Article ID 767328,
2012.Internat. J. Math \& Math Sci, vol. 2012, Article ID 767328,2012

[17] I. S. M. Zawawi, Z. B. Ibrahim, K. I. Othman, Derivation of Diagonally Implicit Block Backward Differentiation Formulas for Solving Stiff Initial Value Problems, Mathematical Problems in Engineering, vol. 2015, Article ID 179231, 2015.

[18] Z. A. Majid, M. B. Suleiman and Z. Omar. 3-Point Implicit Block Method for Solving Ordinary Differential Equations, Bull. Malays. Math. Sci. Soc. (2) 29(1) (2006), pp. 23-31. 2006

[19] R. Allogmany, F. Ismail, Z. B. Ibrahim, Implicit two-point block method with third and fourth derivatives for solving general second order ODEs, Mathematics and Statistics, Vol. 7, No. 4, pp.116-123, 2019 